Revue Française de Civilisation Britannique

\title{
L'Économie d'une Écosse indépendante, de la rente pétrolière au Brexit
}

An Independent Scotland's Economy, from the Oil Windfall to Brexit

\section{Edwige Camp-Pietrain}

\section{OpenEdition}

Journals

Édition électronique

URL : http://journals.openedition.org/rfcb/5045

DOI : $10.4000 /$ rfcb.5045

ISSN : 2429-4373

Éditeur

CRECIB - Centre de recherche et d'études en civilisation britannique

Référence électronique

Edwige Camp-Pietrain, «L'Économie d'une Écosse indépendante, de la rente pétrolière au Brexit », Revue Française de Civilisation Britannique [En ligne], XXIV-4 | 2019, mis en ligne le 25 novembre 2019, consulté le 27 novembre 2019. URL : http://journals.openedition.org/rfcb/5045; DOI : 10.4000/rfcb. 5045

Ce document a été généré automatiquement le 27 novembre 2019.

Revue française de civilisation britannique est mis à disposition selon les termes de la licence Creative Commons Attribution - Pas d'Utilisation Commerciale - Pas de Modification 4.0 International. 


\title{
L'Économie d'une Écosse indépendante, de la rente pétrolière au Brexit
}

\author{
An Independent Scotland's Economy, from the Oil Windfall to Brexit
}

\author{
Edwige Camp-Pietrain
}

\section{Introduction}

1 Depuis 1946, date de publication de la déclaration de politique du SNP (créé en 1934), ses dirigeants présentent des programmes axés sur l'économie, affirmant que les ressources naturelles de l'Écosse doivent être gérées par un État indépendant. À la fin des années soixante, ce raisonnement fut étayé par la découverte d'hydrocarbures en mer du Nord. Le SNP ne cessa de produire des données chiffrées, afin de mettre en évidence les capacités de l'Écosse, par opposition au Royaume-Uni devenu «l'homme malade » de l'Europe ${ }^{1}$.

2 Ces programmes contribuèrent à assurer son succès électoral à la Chambre des Communes (où il obtint 11 des 71 sièges écossais en octobre 1974)². Au Parlement écossais, instauré en 1999, il emporta une majorité relative (2007) puis absolue (2011). Son leader, l'économiste Alex Salmond, devint First Minister. Il s'entoura d'un conseil, composé d'experts de renommée internationale, formulant des avis sur les orientations économiques. Puis des groupes de travail affinèrent son projet indépendantiste, en vue du référendum du 18 septembre $2014^{3}$. Si les unionistes (Travaillistes, Conservateurs et Libéraux-démocrates, opposés à l'indépendance) durent reconnaître que l'économie écossaise ne pouvait plus être considérée comme « dépendante ${ }^{4}$ de son grand voisin, ils mirent en avant certaines faiblesses, suffisantes pour convaincre des indécis de rejeter l'indépendance. Néanmoins, ils acceptèrent de conférer au Parlement écossais des compétences supplémentaires, notamment en matière fiscale (Scotland Act de 2016). 
3 Ces questions institutionnelles, considérées comme réglées, ont resurgi avec le référendum du 23 juin 2016 sur le maintien du Royaume-Uni dans l'UE. Les Écossais s'étant distingués des Anglais et des Gallois en votant contre le Brexit, cela pouvait légitimer l'organisation d'un second référendum d'autodétermination. Afin de compléter son programme économique, Nicola Sturgeon, First Minister depuis novembre 2014, a mis en place une Sustainable Growth Commission (SGC), présidée par Andrew Wilson, un économiste ayant travaillé aux côtés de Salmond ${ }^{5}$. Le rapport de celle-ci, publié en mai 2018, a été approuvé par le SNP en avril 2019.

4 Cependant, dans un contexte d'incertitudes inhérentes au Brexit, il convient de se demander dans quelle mesure la SGC a pu répondre à deux critiques récurrentes en 2014, la viabilité d'une Écosse indépendante et son autonomie réelle. Au-delà, il s'agit de rendre hommage à Jacques Leruez, qui s'est intéressé tout au long de sa carrière aux questions politiques et économiques, sur le plan britannique comme sur le plan écossais ${ }^{6}$.

\section{La viabilité d'une Écosse indépendante}

Le SNP fonde son programme indépendantiste sur les richesses matérielles et humaines de l'Écosse, suffisantes pour équilibrer le budget.

\section{Des atouts matériels et humains à consolider}

6 Les dirigeants du SNP justifient l'accession à l'indépendance par l'existence de ressources génératrices de croissance économique ; dans les années 2000, ils ont ajouté le concept de croissance inclusive, susceptible de bénéficier au plus grand nombre de personnes.

7 D'une part, s'agissant des ressources nationales, le SNP a axé sa campagne de 2014 sur le schéma des années soixante-dix, selon lequel la plupart des hydrocarbures forés en mer $\mathrm{du}$ Nord se trouveraient dans des eaux sous la souveraineté de l'Écosse indépendante, laquelle bénéficierait alors des impôts collectés en sus de l'impôt sur les sociétés auprès des opérateurs privés, détenteurs de licences. Les recettes fiscales (plus de 11 milliards de livres en 2011-2012, tableau 1) représenteraient près de $20 \%$ du budget de l'État indépendant. Une partie d'entre elles (jusqu'à un milliard par an) abonderait un fonds souverain, sur le modèle des conseils des îles Orcades et Shetland. Certes, ces recettes étaient sur le déclin, car les puits rentables étant épuisés, il fallait inciter, par des dégrèvements fiscaux, à la recherche de nouveaux gisements, plus coûteux. Mais le SNP tablait encore sur 6,9 milliards de livres pour 2016-2017, première année de l'État indépendant, soit le double des prévisions du gouvernement britannique.

Or, à la fin de 2014, le cours du baril a chuté de moitié, ce qui a entraîné une hausse des incitations fiscales. Les recettes se sont effondrées, pour s'établir à 36 millions en 2016-2017, un étiage sans précédent depuis 1980. Elles n'excéderaient pas 3 milliards si le baril retrouvait son niveau de 2014 (100 dollars) ${ }^{7}$. La SGC les considère donc comme des revenus exceptionnels, épargnés dans un fonds d'investissement destiné à soutenir la lutte contre les inégalités sociales et la transition climatique. 
9 Reste à développer les ressources renouvelables. En la matière, le SNP continue à se prévaloir d'un potentiel sans égal en Europe, grâce à ses façades maritimes. Les Verts écossais, également indépendantistes, s'en réjouissent, estimant qu'elles peuvent créer plus d'emplois que les hydrocarbures (200 000 contre 156 000), à condition de mobiliser tous les acteurs et les autorités publiques (politique fiscale, prises de participations) ${ }^{8}$.

10 Cependant, des doutes persistent quant à la viabilité financière de ces investissements sans le soutien des infrastructures britanniques ${ }^{9}$ et quant à l'authenticité des intentions au regard du bilan écologique du Parlement écossais. Certes, en 2009, des objectifs de réduction des gaz à effet de serre plus contraignants que ceux du reste du Royaume-Uni ont été fixés ( $42 \%$ d'ici 2020 et $80 \%$ d'ici 2050, par rapport à 1990). Mais avec les compétences conférées par le Scotland Act de 2016, le gouvernement écossais a adopté un simple moratoire sur les gaz de schiste ${ }^{10}$. C'est sous la pression de l'opposition qu'il doit rétrocéder aux communautés côtières les revenus des activités pratiquées jusqu'à 12 milles nautiques du plateau continental (propriétés du Crown Estates), ce qui devrait stimuler les énergies renouvelables. Mais fin avril 2019, la reconnaissance de l'urgence climatique par Sturgeon a semblé marquer une rupture ${ }^{11}$.

11 D'autre part, la SGC promeut une croissance économique inclusive, pour des raisons de justice sociale mais aussi d'efficacité, comme l'a montré Joseph Stiglitz, l'un des conseillers de Salmond ${ }^{12}$. Les élus indépendantistes soulignent les atouts de leur nation : taux de chômage des jeunes en forte régression (de $25 \%$ à 8,4\% fin 2018), taux de chômage plus faible qu'en Angleterre (3,6 \% contre $4 \%$ ), inégalités sociales un peu moins marquées (coefficient de Gini). Mais les Conservateurs rappellent que sa croissance est inférieure d'un tiers à la croissance britannique depuis $2007^{13}$, sans perspective d'amélioration (tableau 2).

12 L'une des priorités de la SGC est de stimuler la productivité, problème récurrent de l'économie écossaise ${ }^{14}$. Comme le SNP en 2014, la SGC met l'accent sur l'enseignement, en s'appuyant sur l'excellence des universités locales (source de recherches et d'innovations, en relation avec les entreprises) et sur le potentiel de chaque individu ${ }^{15}$. Elle veut stimuler le travail des femmes. Par ailleurs, elle préconise la flexisécurité du marché du travail, associant flexibilité et accompagnement actif dans la recherche d'emploi.

Ces propositions pourraient nécessiter des réorientations des politiques des gouvernements SNP. En effet, ceux-ci ont pris des mesures pour faciliter le travail des femmes, tout en réduisant les budgets de l'enseignement professionnel. Dans le champ restreint de leurs compétences en matière sociale, ils ont atténué les effets de la flexibilité britannique, pour le salaire minimum et la saisie des tribunaux des prud'hommes ${ }^{16}$. Mais ils ont également renforcé l'accompagnement des chômeurs au lieu de les sanctionner.

14 Par ailleurs, d'aucuns regrettent le conservatisme de ces recommandations, s'agissant autant de la structure et du fonctionnement des entreprises privées (confédération syndicale STUC ${ }^{17}$ ), que de la nature des emplois existants face au développement de l'intelligence artificielle (George Kerevan, ex-député SNP) ${ }^{18}$.

\section{La recherche de l'équilibre budgétaire}

15 En 2014, les dirigeants du SNP anticipaient un déficit budgétaire, guère supérieur aux déficits britanniques (tableaux 3 et 4). Ils misaient sur de nouvelles recettes (pétrole) et 
sur des économies (nucléaire, administration). Ils restaient imprécis sur le plan fiscal, sinon pour abaisser l'impôt sur les sociétés au-dessous du taux britannique afin d'attirer les entreprises. Quant aux dépenses, ils avaient une estimation optimiste des coûts inhérents à la constitution d'un État (450 milliards). Ils promettaient de maintenir les acquis sociaux et même de revenir aux fondements de l'État-Providence de l'après-guerre, car la fragilisation de ces derniers par les gouvernements britanniques depuis 1979 affectait l'Écosse, même dans les domaines dévolus. Cet argumentaire a été entendu, le système de santé étant l'une des principales préoccupations des électeurs indépendantistes ${ }^{19}$.

Cependant, au cours des années suivantes, l'écart entre les données écossaises et britanniques s'est creusé. En 2016-2017, le «déficit » de l'Écosse s'est établi à 8,9\%, environ deux fois plus que prévu en 2014, tandis que le budget britannique tendait vers l'équilibre. Cela est en partie lié à une volonté, partagée par les élus écossais, de limiter les effets des politiques britanniques d'austérité par des hausses relatives de dépenses publiques (tableau 5) assurant la gratuité de services publics et la revalorisation de certaines allocations ${ }^{20}$. Mais les recettes sont sources de divisions. Les Conservateurs sont arc-boutés sur la baisse de la pression fiscale, tandis que les autres partis ne parviennent pas à s'accorder sur le degré de progressivité, seuls les Verts finissant par voter le budget du gouvernement SNP (minoritaire depuis 2016) ${ }^{21}$.

L'indépendance offrirait plus de latitude, à condition de dépasser le clivage entre indépendantistes et unionistes. La SGC prévoit un retour à un déficit inférieur à $3 \% \mathrm{du}$ PIB sur une dizaine d'années, fondé sur une hausse réelle des dépenses publiques inférieure de $1 \%$ au taux de croissance ${ }^{22}$. Elle souligne les finalités à atteindre (lutte contre la pauvreté) sans s'attarder sur les moyens (sinon pour entériner le refus, par Sturgeon, d'accorder une baisse générale de l'impôt sur les sociétés). Évitant tout arbitrage $^{23}$, elle préfère mettre en cause les effets des politiques britanniques.

Les indépendantistes radicaux y ont vu un projet d'austérité, axé sur des équilibres d'inspiration néolibérale, et sur les intérêts des entreprises, que Wilson s'attache à défendre dans son cabinet privé ${ }^{24}$. Ils réclament des politiques susceptibles de transformer la société (Verts), porteuses d'ambitions sociales (Common Weal) ${ }^{25}$.

Les dirigeants SNP ont alors nuancé leur position, évoquant une révision des dépenses (Sturgeon), voire une politique de relance (Derek MacKay, ministre des Finances) ${ }^{26}$, avant de s'engager à créer une commission sur la justice sociale et l'équité (Sturgeon) lors de l'adoption du rapport de la SGC par le SNP ${ }^{27}$. Outre la viabilité du nouvel État se pose la question de sa capacité à mettre en œuvre des politiques indépendantes.

\section{L'autonomie réelle d'une Écosse indépendante}

L'accession à l'indépendance doit permettre la prise de décisions distinctes par des élus écossais. En 2014, à la surprise générale, c'est autour de la monnaie que les passions se sont cristallisées. En 2018, la SGC a mis l'accent sur la capacité d'action des petits États, sans parvenir à faire taire la polémique relative à la devise. Elle avait confiance dans la capacité d'insertion de l'Écosse dans l'économie-monde, malgré le Brexit. 


\section{La monnaie, un symbole de l'indépendance}

21 Le SNP n'avait pas mené de réflexions approfondies sur la monnaie. Les Écossais ayant renoncé à leur monnaie en signant le traité d'Union de 1707, la création d'une devise semblait devoir accompagner l'accession à l'indépendance. Dans les années quatrevingt-dix, le SNP opta pour l'adhésion à l'euro, assortie de conditions restrictives (examen de la conjoncture, consultation de la population), avant d'y renoncer lors de la crise de la zone euro.

Pendant la campagne référendaire, sur les conseils de son groupe d'experts, le gouvernement écossais s'engagea à conserver la livre sterling dans le cadre d'une union monétaire avec le reste du Royaume-Uni. Mais il se heurta au refus de George Osborne, Chancelier de l'Échiquier, des autres unionistes (Libéraux-démocrates et Travaillistes) et même de hauts fonctionnaires ${ }^{28}$. Cette fermeté contribua à décrédibiliser le projet indépendantiste. La monnaie apparut comme le principal facteur motivant un rejet de ce dernier ${ }^{29}$.

23 La SGC a tenu compte de ces arguments, tant pour la monnaie (elle préconise l'utilisation de la livre sterling, sans union monétaire) que pour d'autres aspects de la politique financière.

24 Tout d'abord, s'agissant des opérateurs financiers, elle prône la création d'une Banque centrale écossaise sur le modèle de la Banque d'Angleterre, afin d'assurer la réglementation du système bancaire et d'agir comme prêteur en dernier ressort. En 2014, les unionistes soulignaient qu'une telle institution n'aurait pu assurer le sauvetage de la RBS et de la Bank of Scotland (devenue HBoS) en 2009. Ils martelaient que les fleurons du secteur bancaire écossais, réalisant l'essentiel de leurs activités à l'extérieur de l'Écosse, envisageaient une délocalisation de leur siège en cas d'indépendance. Cette projection, alors contestée par le SNP, a été entérinée par la SGC. De ce fait, la Banque centrale écossaise limiterait son aide aux activités bancaires de détail des filiales installées en Écosse ${ }^{30}$. Mais la SGC ne remet en cause ni le démantèlement $\mathrm{du}$ secteur financier écossais, ni la nature de la réglementation financière ${ }^{31}$.

25 Ensuite, s'agissant des taux d'intérêt, la fixation du taux directeur continuerait à relever de la banque d'Angleterre. En 2014, les indépendantistes affirmaient que celleci tiendrait compte de la conjoncture écossaise. Cet espoir n'est plus de mise en l'absence d'union monétaire. De même, en 2014, les indépendantistes ne pensaient pas que les marchés financiers puissent réclamer des taux élevés au gouvernement écossais, en tant que nouvel acteur. Mais la SGC s'est rangée aux arguments des unionistes, évoquant toutefois un surcoût, inférieur à un point, qui devrait s'estomper $^{32}$, et ne devrait pas se répercuter sur les emprunts contractés par les ménages et les entreprises car ils ne présenteraient pas de risque. Mais selon les unionistes, l'impact du surcoût sur l'économie réelle pourrait atteindre $2 \%$ du PIB sur le court-terme ${ }^{33}$.

26 Enfin, en ce qui concerne les possibilités d'évolution, la SGC n'écartait pas la création d'une monnaie, seul gage d'une véritable indépendance selon de nombreux partisans de celle-ci. Le recours à la livre sterling serait limité à une phase de transition, destinée à assurer la stabilité de l'État indépendant ${ }^{34}$. Mais la SGC proposait six critères d'évaluation, de nature économique (déficits publics et endettement, crédibilité de la Banque centrale écossaise, réserves de change, confiance des ménages et des 
entreprises, structure commerciale de l'économie écossaise, corrélation des cycles économiques de l'Écosse avec ceux de ses principaux partenaires ${ }^{35}$ ). Ce procédé ressemblait à celui employé par Gordon Brown en tant que Chancelier de l'Échiquier s'agissant de l'euro, pour se ménager une décision politique permettant d'enterrer le projet d'adhésion en 2003.

Cependant, cette option était controversée au sein du mouvement indépendantiste. Dès 2017, Common Weal a publié une analyse détaillée concluant que l'utilisation de la monnaie d'un autre État sans union monétaire n'était réaliste que pour des micro-États tels Andorre ou le Liechsteintein, alors que l'Écosse, qui représentait $10 \%$ de l'économie britannique, avait des intérêts propres ${ }^{36}$. Seule une devise écossaise garantirait la souveraineté, en restant arrimée à la livre sterling dans un premier temps, avant de pouvoir éventuellement s'en démarquer.

La polémique ayant pris de l'ampleur lors des assemblées consultatives organisées par le SNP, en mars 2019, Keith Brown, numéro deux du parti, et Derek MacKay ont précisé que la livre ne serait conservée que dans une période transitoire, une devise étant créée dès que possible, grâce au redressement de l'économie. Mais nombre d'indépendantistes, qui exigeaient une transition plus rapide et l'abandon des six critères $^{37}$, ont obtenu satisfaction sur le premier point. En effet, à l'issue d'une campagne menée par Kerevan et Ronnie Cowan, député à la Chambre des Communes, le Congrès du SNP, réuni en avril 2019, s'est engagé à préparer l'adoption d'une devise si les Écossais se prononçaient en faveur de l'indépendance ${ }^{38}$.

Cette clarification, indispensable pour de nombreux indépendantistes, ne sert pas nécessairement leur cause, l'opinion publique restant attachée à la livre sterling sur le long terme. La proportion atteignait deux-tiers en 2016 selon BMG (24\% pouvant envisager une devise écossaise), $47 \%$ au début de 2019 (23\% approuvant la transition prônée par la SGC, 6 \% réclamant une évolution plus rapide) ${ }^{39}$. En tout cas, l'euro était plébiscité par moins d'un sondé sur huit, même si cette option ne saurait être écartée selon les unionistes, car la promesse d'intégration de la monnaie unique est exigée des candidats à l'adhésion à l'UE.

\section{La petite taille, un atout}

30 En 2014, les références du SNP aux petits États étaient ponctuelles. À son arrivée au pouvoir, en 2007, Salmond avait érigé en modèle l'arc de prospérité allant de la République d'Irlande aux États scandinaves. Lorsque la crise financière de 2008 mit en évidence leur fragilité, le First minister continua à louer la réactivité des petits États (le gouvernement irlandais face à ses banques), et leur capacité à rechercher des compromis par la discussion (notamment au sein de l'UE, face aux grands États). Les dirigeants du SNP mettaient aussi en avant les politiques redistributives des pays scandinaves, mais ils préféraient s'attarder sur le volet dépenses que sur des prélèvements sociaux plus élevés qu'en Écosse. Les unionistes anticipaient, à l'inverse, un affaiblissement de la voix écossaise et de sa capacité à défendre ses intérêts au sein de l'UE.

31 À l'issue du référendum, le gouvernement écossais est resté centré sur des modèles européens. Il entend poursuivre le rapprochement avec les pays nordiques, auquel il associe désormais les pays baltes ${ }^{40}$. 
32 La SGC a élargi ses références, englobant les petits États (entre 5 et 10 millions d'habitants) hors de l'UE, en Europe (Suisse) ou ailleurs (Singapour, Nouvelle-Zélande, et même Hong-Kong). Elle met en avant leurs atouts par rapport aux grands États : croissances démographique et économique, PIB par habitant, taux de chômage, efficacité de l'action gouvernementale, part des exportations et des investissements directs de l'étranger (IDE) rapportée au PIB, investissement soutenu dans le capital humain ${ }^{41}$. Si les bilans en matière de productivité, d'inégalités de revenus entre classes et sexes, de recherche et développement, de dépenses publiques sont plus diversifiés, la SGC souligne que ces États mènent des politiques distinctes. Leurs emprunts ne sont pas plus coûteux, comme en témoignent les taux d'intérêts sur les obligations à dix ans.

33 Selon la SGC, au sein du Royaume-Uni, les dirigeants écossais sont empêchés de prendre des initiatives susceptibles de stimuler le potentiel économique ${ }^{42}$. Ainsi, le gouvernement britannique a gaspillé les recettes pétrolières (328 milliards) en dépenses courantes, alors que son homologue norvégien a constitué un fonds atteignant 750 milliards d'euros. De plus, une réduction de l'écart salarial hommesfemmes pourrait entraîner une hausse du PIB comprise entre 800 millions (modèle suédois) et 6 milliards (modèle néo-zélandais).

34 L'Écosse doit se doter d'une stratégie nationale s'inspirant des meilleurs exemples étrangers; elle doit viser une croissance égale ou supérieure à celle des petits États (2,5\% puis 3,5\%), plus ambitieuse que les prévisions disponibles (tableau 2). Cette croissance doit profiter à l'ensemble de la population. Elle peut s'accompagner d'une flexibilité du marché du travail (modèle danois), tout en sécurisant la protection sociale fragilisée par les réformes britanniques. Les entreprises doivent être soutenues, pour leurs investissements, leurs exportations, leurs activités quotidiennes (plan d'action irlandais) afin d'améliorer la productivité (commission ad hoc néo-zélandaise). Mais le dumping fiscal irlandais est écartét ${ }^{43}$.

Or, ces choix restent sélectifs. Kerevan, indépendantiste, dénonce l'attention portée à trois États, Finlande, Danemark et Nouvelle-Zélande, pour l'innovation et les ressources naturelles, tout en occultant les aspects en contradiction avec les valeurs du SNP (respectivement austérité, montée de l'extrême-droite, néolibéralisme).

36 Les unionistes s'étonnent de l'absence du Portugal ou de la Grèce, tout en soulignant que les performances des "modèles" ne se démarquent pas tellement de celles du Royaume-Uni ${ }^{44}$. Ils rappellent, comme en 2014 , la capacité des grands États à absorber les chocs. Les Travaillistes contrastent l'austérité proposée par la SGC, qu'ils surnomment commission « des coupes budgétaires » (fort éloignée de la lutte contre les inégalités, érigée en priorité par Sturgeon) et les politiques scandinaves qui se rapprochent de certaines de leurs propositions pour le Royaume-Uni ${ }^{45}$. Enfin, la SGC sous-estime les contraintes pesant sur des décisions prises par un nombre plus réduit de personnes, dans un petit État, sous la pression d'un grand voisin ${ }^{46}$.

\section{L'insertion dans l'économie-monde en dépit du Brexit}

Le SNP conçoit l'indépendance comme un moyen pour l'Écosse de s'insérer dans l'économie mondiale. Les propositions de la SGC, optimistes, ont été éclipsées par les polémiques engendrées par le Brexit.

38 S'agissant du commerce, la SGC reprend la stratégie du SNP en 2014, cherchant à encourager les acteurs à travailler de concert (team Scotland) et à créer un réseau de 
correspondants, afin de tirer parti de la marque Écosse et ses avantages concurrentiels ${ }^{47}$. Elle affiche des objectifs volontaristes (création d'une commission de la productivité) et des moyens moins agressifs (l'impôt devant favoriser les pratiques vertueuses).

En ce qui concerne l'immigration, en 2014, le SNP entendait favoriser l'accueil et l'insertion de toutes les catégories d'immigrés, soulignant sa conception ouverte de la citoyenneté (droit du sol). Mais il n'écartait pas un système de points pour l'immigration économique. De même, la SGC préconise la simplification des formalités et des incitations fiscales pour attirer des immigrés du monde entier, dénonçant, comme les élus écossais ${ }^{48}$, des politiques britanniques restrictives, peu adaptées aux besoins locaux.

40 Mais le débat public s'est focalisé sur l'UE. Nicola Sturgeon s'est prononcée dès 2016 en faveur de l'Espace économique européen (EEE), permettant de rester dans le marché unique, renonçant si nécessaire à l'union douanière, sur le modèle norvégien. Ce compromis serait moins pénalisant pour l'économie écossaise que l'Association européenne de libre-échange (AELE, accords avec l'UE au cas par cas) ou qu'une sortie sans accord, conduisant à un commerce aux conditions de l'OMC (tableau 6) ${ }^{49}$. En effet, $56 \%$ des exportations écossaises sont liées à l'UE (43\% directement, $13 \%$ par les accords de libre-échange avec des pays tiers). Certaines de ces exportations émanent de sociétés étrangères tirant avantage du marché unique. Ces IDE représentent 2350 entreprises (dont $44 \%$ originaires de l'UE) et 318000 emplois.

41 De plus, en 2017, l'Écosse comptait 219000 citoyens de l'UE (soit deux-tiers des ressortissants étrangers), parmi lesquels près de 140000 en provenance des États devenus membres en 2004 ; cette population est jeune et active (60\% ont moins de 35 ans, $77 \%$ ont un emploi, contre $42 \%$ et $73 \%$ de l'ensemble des résidents). Selon les modélisations du gouvernement écossais ${ }^{50}$, chaque ressortissant de l'UE rapporte en moyenne 10400 livres en recettes publiques et 34400 livres au PIB. Seuls ces flux en provenance de l'UE peuvent permettre à l'Écosse (moins attractive auprès du reste du monde) de rattraper son retard sur l'ensemble du Royaume-Uni en matière de croissance du PIB (ce facteur étant aussi important que l'évolution du chômage, tableau 7) et de mettre un terme au déclin de sa population (dont le solde naturel est négatif).

Le gouvernement écossais s'est donc indigné des options retenues par le gouvernement britannique de Theresa May (sortie du marché unique et de l'union douanière), mais aussi de l'acceptation, dans l'attente d'un accord de libre-échange consécutif au Brexit, du maintien de l'Irlande du Nord dans le marché unique. L'Écosse est exclue de ce traitement différencié ${ }^{51}$. De plus, le gouvernement britannique a invité son homologue écossais à mener des politiques stimulant commerce et immigration dans son champ de compétences, sans élargir ce dernier ${ }^{52}$.

43 Cette intransigeance conforte la nécessité d'accéder à l'indépendance au sein de l'UE, car celle-ci défend ses membres de petite taille, notamment l'Irlande sur la question de la frontière. Le gouvernement britannique, qui a ignoré les intérêts écossais, ne peut se prévaloir d'offrir un cadre protecteur, comme en 2014.

De ce fait, le gouvernement écossais entend promouvoir l'image de sa nation, ouverte sur le monde, par opposition à une Angleterre qui se replie. Il martèle que les ressortissants de l'UE sont bienvenus. Il mène, en collaboration avec les agences Scottish 
Enterprise, Scottish Development International, VisitScotland, les universités, une campagne de promotion de l'Écosse auprès des touristes, des étudiants et des investisseurs ${ }^{53}$.

Il a publié une stratégie commerciale visant à faire passer la part des exportations dans le PIB écossais de $20 \%$ à $25 \%$ sur dix ans ${ }^{54}$. D'une part, il agit auprès des principaux partenaires de l'Écosse, en désignant un ministre du Commerce, en ouvrant des bureaux (Dublin, Francfort, Paris, Ottawa) et en mobilisant diaspora et envoyés spéciaux (telle Wendy Alexander, ancienne ministre travailliste). D'autre part, il souhaite aider les entreprises. Une banque nationale d'investissement va compléter les subventions aux entreprises, sans condition de nationalité ${ }^{55}$. Les PME, déjà dispensées de taxe locale, vont bénéficier d'un fonds de soutien à l'exportation, grâce au parrainage de sociétés plus expérimentées (avec CBI Scotland).

Les unionistes craignent la marginalisation d'un petit État indépendant, mais les termes du débat ont évolué depuis 2014. S'agissant du commerce, le coût d'une rupture avec le principal partenaire (britannique) de l'Écosse serait huit fois supérieur à celui d'une sortie de l'UE sans accord ${ }^{56}$, alors que celle-ci est considérée comme la pire des hypothèses (tableau 6), tant pour les grandes villes que pour les périphéries rurales ${ }^{57}$. Quant à la libre circulation, les unionistes s'interrogeaient en 2014 sur la possibilité de maintenir une Écosse indépendante dans la common travel area avec le reste du Royaume-Uni et l'Irlande. Cette position est difficile à justifier en 2019, pour une frontière courte ( $70 \mathrm{~km}$, huit points de passage) et pacifiée depuis des siècles ${ }^{58}$ alors que des dérogations sont envisagées pour l'Irlande du Nord à l'issue du Brexit.

Enfin, l'opinion publique est plus réservée que le gouvernement écossais. En 2017, selon Panelbase, seuls $45 \%$ des sondés estimaient que les ressortissants de l'UE devraient conserver le droit de vivre et travailler au Royaume-Uni, $38 \%$ y étant opposés. D'après l'enquête sociologique annuelle Scottish social attitudes, si les Écossais souhaitent maintenir le libre-échange (90\%), $63 \%$ accepteraient pour cela la libre circulation des personnes. Mais deux-tiers prônent les mêmes règles de commerce et d'immigration pour le Royaume-Uni.

\section{Conclusion}

Le SNP, qui a fondé son nationalisme sur les questions économiques, et sur lequel un économiste, Salmond, a imprimé sa marque, semblait armé pour répondre aux interrogations dans ce domaine. Pourtant, en 2012 comme en 2016, ses dirigeants ont eu recours à des experts pour conforter leur crédibilité. De plus, la SGC a vu ses propositions marginalisées par un Brexit susceptible d'affecter la viabilité et l'autonomie de l'Écosse.

D'une part, depuis 2016, le gouvernement SNP, qui peine à s'affranchir des comparaisons avec l'Angleterre, est focalisé sur les bienfaits de l'UE sur le plan économique, multipliant les projections détaillées (malgré leur caractère hypothétique). La SGC, qui a une vision plus large, veut améliorer l'insertion de l'Écosse dans l'économie-monde, pour faire face à tous les scénarios. Mais la réalisation de son projet dépend en partie d'un Brexit qui échappe au gouvernement écossais.

50 D'autre part, en 2018, lors de la publication du rapport, la pêche, qui n'est pas mentionnée dans ce dernier, a dominé les débats parlementaires, pour des raisons politiques (SNP et Conservateurs se trouvant en concurrence auprès des communautés 
côtières). Or, les pêcheurs, qui veulent quitter la politique commune de la pêche, craignent que le gouvernement britannique n'autorise les bateaux de l'UE à pêcher dans leur zone économique exclusive en échange d'un accès au marché unique (satisfaisant l'industrie agro-alimentaire) ${ }^{59}$.

51 En outre, le Brexit accentue le dilemme entre deux unions. Les incertitudes économiques constituaient déjà le premier facteur de rejet de l'indépendance en $2014^{60}$, alors que le marché unique devait permettre le maintien de liens étroits. Pis, le Brexit engendre de nouvelles fractures, entre des ouvriers plus eurosceptiques mais toujours plus enclins à voter pour l'indépendance que les classes moyennes pourtant attachées à $\mathrm{I}^{\prime} \mathrm{UE}^{61}$. D'ailleurs, prenant acte de la régression du SNP aux élections à la Chambre des Communes de juin 2017, Sturgeon n'a cessé de repousser la requête d'un second référendum d'autodétermination, tout en évitant d'évoquer les recommandations de la SGC, d'autant que les contradictions du processus de Brexit contrariaient les promesses de continuité en cas d'indépendance.

52 Les reports successifs du Brexit, à l'issue du 29 mars 2019, ont changé la donne. Le 23 avril, Sturgeon a demandé un second référendum d'autodétermination d'ici la fin de la législature écossaise (2021), tout en soulignant la nécessité de forger un consensus autour de l'indépendance, avec l'appui de Wilson, et d'Angus Robertson, son ancien numéro deux qui, après sa défaite en 2017, a créé un institut d'études (Progress Scotland). Elle entend mettre l'accent sur l'économie, en diffusant le projet de la SGC et en le consolidant (notamment sur le plan social), tout en affirmant l'importance de l'UE $^{62}$. Elle a annoncé la mise en place d'assemblées citoyennes pour discuter de l'avenir de l'Écosse. Elle a vu dans le succès du SNP aux élections au Parlement européen du 23 mai 2019 une nouvelle dynamique. Mais Sturgeon devra composer avec des voix divergentes au sein du mouvement indépendantiste qui, loin de se satisfaire de leur victoire partielle sur la question de la monnaie, exigent un changement réel d'orientation et une accélération du calendrier ${ }^{63}$, au lieu de se perdre dans des conjectures autour d'un second référendum sur le Brexit.

Edwige Camp-Pietrain est Professeur de civilisation britannique à l'Université Polytechnique des Hauts-de-France (Valenciennes). Membre du laboratoire CALHISTE EA4343, ses recherches portent sur la vie politique, les institutions politiques et les politiques publiques dans l'Écosse contemporaine.

Annexes

Tableau 1 : Évolution des recettes fiscales britanniques liées au pétrole en mer du Nord

\begin{tabular}{|l|l|}
\hline Année fiscale & Recettes en milliards de livres sterling \\
\hline $1980-1981$ & 3,861 \\
\hline $1981-1982$ & 6,395 \\
\hline $1982-1983$ & 7,867 \\
\hline $1983-1984$ & 8,788 \\
\hline $1984-1985$ & 12,326 \\
\hline
\end{tabular}




\begin{tabular}{|c|c|}
\hline $1985-1986$ & 11,217 \\
\hline $1986-1987$ & 4,701 \\
\hline $1987-1988$ & 4,736 \\
\hline 1988-1989 & 3,301 \\
\hline $1989-1990$ & 2,502 \\
\hline 1990-1991 & 2,342 \\
\hline 1991-1992 & 1,017 \\
\hline $1992-1993$ & 1,338 \\
\hline 1993-1994 & 1,266 \\
\hline 1994-1995 & 1,673 \\
\hline $1995-1996$ & 2,341 \\
\hline 1996-1997 & 3,351 \\
\hline 1997-1998 & 3,33 \\
\hline 1998-1999 & 2,511 \\
\hline $1999-2000$ & 2,564 \\
\hline $2000-2001$ & 4,455 \\
\hline $2001-2002$ & 5,426 \\
\hline $2002-2003$ & 5,097 \\
\hline $2003-2004$ & 4,284 \\
\hline $2004-2005$ & 5,183 \\
\hline $2005-2006$ & 9,384 \\
\hline $2006-2007$ & 8,924 \\
\hline $2007-2008$ & 7,474 \\
\hline 2008-2009 & 12,456 \\
\hline $2009-2010$ & 5,991 \\
\hline $2010-2011$ & 8,406 \\
\hline 2011-2012 & 11,336 \\
\hline
\end{tabular}




\begin{tabular}{|l|l|}
\hline $2012-2013$ & 6,632 \\
\hline $2013-2014$ & 4,499 \\
\hline $2014-2015$ & 1,691 \\
\hline $2015-2016$ & $-0,085$ \\
\hline $2016-2017$ & 0,036 \\
\hline $2017-2018$ & 1,192 \\
\hline
\end{tabular}

Tableau 2 : Prévisions de l'évolution des fondamentaux des économies britannique et écossaise selon les organismes indépendants Office for Budget Responsibility (OBR, mars 2018) et Scottish Fiscal Commission (SFC, mai 2018)

\begin{tabular}{|c|c|c|c|c|c|c|c|c|}
\hline & & 2017 (données) & 2018 & 2019 & 2020 & 2021 & 2022 & 2023 \\
\hline \multirow[t]{2}{*}{ PIB } & OBR & 1,7 & 1,5 & 1,3 & 1,3 & 1,4 & 1,5 & \\
\hline & SFC & 0,8 & 0,7 & 0,8 & 0,9 & 0,9 & 0,9 & 0,9 \\
\hline \multirow[t]{2}{*}{ PIB par habitant } & OBR & 1,1 & 0,9 & 0,7 & 0,7 & 0,8 & 0,9 & \\
\hline & SFC & 0,2 & 0,3 & 0,5 & 0,6 & 0,6 & 0,7 & 0,7 \\
\hline \multirow[t]{2}{*}{ Emploi (millions) } & OBR & 32,1 & 32,2 & 32,4 & 32,5 & 32,6 & 32,7 & \\
\hline & SFC & 2,6 & 2,6 & 2,6 & 2,7 & 2,7 & 2,7 & 2,7 \\
\hline \multirow[t]{2}{*}{ Salaires réels } & OBR & 0,5 & 0,5 & 0,6 & 0,6 & 0,8 & 1 & \\
\hline & SFC & -1 & $-0,5$ & 0 & 0,2 & 0,6 & 0,9 & 1,2 \\
\hline \multirow[t]{2}{*}{ Gains nominaux } & OBR & 2,6 & 2,7 & 2,4 & 2,5 & 2,8 & 3 & \\
\hline & SFC & 1,1 & 1,6 & 1,9 & 2,2 & 2,6 & 2,9 & 3,2 \\
\hline
\end{tabular}

Tableau 3 : Prévisions des déficits publics en proportion du PIB à la fin de 2013 pour 2016-2017

\begin{tabular}{|l|l|l|l|l|}
\hline & $\begin{array}{l}\text { Royaume-Uni } \\
(\mathrm{OBR})\end{array}$ & $\begin{array}{l}\text { Royaume-Uni } \\
\text { sans Écosse }\end{array}$ & $\begin{array}{l}\text { Écosse (gouvernement } \\
\text { écossais) }\end{array}$ & $\begin{array}{l}\text { Écosse (fourchette basse } \\
\text { revenus pétrole) }\end{array}$ \\
\hline $\begin{array}{l}\text { Déficits } \\
\text { publics }\end{array}$ & $2,6 \%$ & $2,3 \%$ & $3,2 \%$ & $5,3 \%$ \\
\hline
\end{tabular}


Tableau 4 : Évolution des déficits publics en proportion du PIB

\begin{tabular}{|c|c|c|c|}
\hline & Écosse sans pétrole & Écosse avec pétrole & Royaume-Uni \\
\hline $2005-2006$ & 10,1 & 1,7 & 2,9 \\
\hline 2006-2007 & 9,8 & 2,3 & 2,3 \\
\hline $2007-2008$ & 9,4 & 2,6 & 2,4 \\
\hline 2008-2009 & 13,7 & 2,9 & 6,7 \\
\hline $2009-2010$ & 17,8 & 10,6 & 11,1 \\
\hline $2010-2011$ & 16,1 & 8,5 & 9,3 \\
\hline 2011-2012 & 14,7 & 5,8 & 7,6 \\
\hline $2012-2013$ & 14 & 8,3 & 7,3 \\
\hline $2013-2014$ & 11,9 & 8,3 & 5,5 \\
\hline 2014-2015 & 10,7 & 8,9 & 4,9 \\
\hline $2015-2016$ & 10,5 & 9,7 & 3,8 \\
\hline 2016-2017 & 9,7 & 8,9 & 2,3 \\
\hline $2017-2018$ & 9,5 & 7,9 & 1,9 \\
\hline
\end{tabular}

Tableau 5 : Évolution des dépenses publiques totales par habitant (en livres sterling)

\begin{tabular}{|l|l|l|l|}
\hline Année fiscale & Royaume-Uni & Écosse & Écart Écosse/Royaume-Uni (en \%) \\
\hline $2008-2009$ & 10263 & 11408 & 11,1 \\
\hline $2009-2010$ & 10796 & 11852 & 10,98 \\
\hline $2010-2011$ & 11046 & 12158 & 11,01 \\
\hline $2011-2012$ & 10951 & 12227 & 11,16 \\
\hline $2012-2013$ & 10998 & 12265 & 11,15 \\
\hline $2013-2014$ & 11425 & 12708 & 11,2 \\
\hline $2014-2015$ & 11594 & 12821 & 10 \\
\hline $2015-2016$ & 11599 & 12914 & 11,3 \\
\hline $2016-2017$ & 11742 & 13190 & 12,3 \\
\hline
\end{tabular}




\begin{tabular}{|l|l|l|l|}
\hline $2017-2018$ & 11954 & 13530 & 13,2 \\
\hline
\end{tabular}

Tableau 6 : Modélisation de l'impact du Brexit sur les fondamentaux de l'économie écossaise en dix ans

\begin{tabular}{|l|l|l|l|}
\hline & EEE & AELE & OMC \\
\hline Exportations de biens & -12 à $-18 \%$ & -12 à $-18 \%$ & $-26 \%$ \\
\hline Exportations de services & -11 à -18 \% & -18 à -22 \% & $-25 \%$ \\
\hline Croissance du PIB (\%) & -2 à $3 \%$ & -3 à $4 \%$ & $-5 \%$ \\
\hline PIB par rapport à 2015-2016 (livres) & -3 à 5 milliards & -4 à 6 milliards & -8 milliards \\
\hline Croissance des salaires réels (\%) & -3 à $4 \%$ & -5 à $6 \%$ & $-7 \%$ \\
\hline Salaires réels, travail à temps plein (livres) & -800 à 1200 & -1200 à 1600 & -2000 \\
\hline Taux d'emplois & -1 à $2 \%$ & -1 à $2 \%$ & $-3 \%$ \\
\hline Nombre d'emplois & -30 000 & -30 000 & -80000 \\
\hline
\end{tabular}

Tableau 7 : Prévisions de l'évolution des fondamentaux de l'économie écossaise : effet de différents facteurs

\begin{tabular}{|l|l|l|l|l|l|}
\hline & $\begin{array}{l}\text { Prévision } \\
\text { moyenne } \\
2018-2023\end{array}$ & $\begin{array}{l}\text { Variations selon } \\
\text { les flux } \\
\text { migratoires }\end{array}$ & $\begin{array}{l}\text { Variations } \\
\text { selon le taux de } \\
\text { chômage }\end{array}$ & $\begin{array}{l}\text { Variations selon } \\
\text { le nombre } \\
\text { d'heures } \\
\text { travaillées }\end{array}$ & $\begin{array}{l}\text { Variations selon } \\
\text { la productivité }\end{array}$ \\
\hline PIB & 0,9 & 0,7 à 0,9 & 0,8 à 1 & 0,7 à 1,1 & 0,4 à 1,5 \\
\hline Emploi & 0,1 & $-0,1$ à 0,2 & 0 à 0,2 & 0,1 & 0,1 \\
\hline $\begin{array}{l}\text { Gains } \\
\text { moyens }\end{array}$ & 2,5 & 2,5 & 2,5 & 2,3 à 2,7 & 2,1 à 3,1 \\
\hline
\end{tabular}

\section{BIBLIOGRAPHIE}

Alston Philip, Statement, Londres, novembre 2018. 
Bell David, « The Contemporary Landscape », in Gibb Kenneth, MacLennan Duncan, McNulty Des, Comerford Michael (dir), The Scottish Economy, Londres, Routledge, 2017, p. 26-39.

Bella Caledonia, Replying to Andrew Wilson : a Scottish Currency is not Symbolic, it's Power, 14 février 2019, bellacaledonia.org.uk/2019/02/14/replying-to-andrew-wilson-a-scottish-currency-is-notsymbolic-its-power/, consulté le 20 février 2019.

Brooks Lilly, «SNP Votes for Scotland to Quickly Adopt New Currency if it Leaves the UK », The Guardian, 27 avril 2019.

Carpenter Griffin, "Troubled Waters, Scottish Fisheries and the EU », in Hassan Gerry, Gunson Russell (dir.), Scotland, the UK and Brexit. A Guide to the Future, Édimbourg, Luath Press, 2017, p. 72-77.

common weal, White Paper Project. A Business Plan for a New Nation, Édimbourg, 2018.

Curtice John, Does the Scottish Growth Commission Matter ?, 24 mai 2018, www.whatscotlandthinks.org, consulté le 30 juin 2018.

Dalzell Craig, Scottish Currency Options post-Brexit. A Discussion Paper, Common Weal, juillet 2016 (allofusfirst.org/policy), consulté le 30 avril 2017.

Dalzell Craig, The Demographics of Independence, Common Weal, juin 2018 (allofusfirst.org/policy), consulté le $1^{\mathrm{er}}$ juillet 2018.

Dalzell Craig, "Scotland Deserves More than Trap of 'Soft Independence' », The Herald, ${ }^{\mathrm{er}}$ mai 2019 , p. 20.

Dhingra Swati, Machin Stephen, Overman Henry, The Local Economic Effects of Brexit, Londres, London School of Economics, Centre for Economic Performance, paper 10, 2017.

EY, Sectoral Impact Analysis and British Readiness Assessment, Édimbourg, Scottish Government, janvier 2019.

Fiscal Commission Working Group, The Economic Foundations of an Independent Scotland, Édimbourg, Scottish Government, août 2014.

Fraser of Allander Institute, Long-Term Economic Implications of Brexit, octobre 2016.

Gordon Tom, « Sturgeon Planning IndyRef2 Vote Before 2021 Ballot », The Herald, 25 avril 2019, p. 1.

Goudie Andrew (dir.), Scotland's Future. The Economics of Constitutional Change, Dundee, Dundee University Press, 2013.

Grant Alistair, « Row over Nats Plans for New Currency in an Independent Scotland », The Herald, 5 mars 2019, p. 6.

Grant Alistair, «SNP Needs to Have Post-Brexit Answers Ahead of Indy Ref2 », The Herald, 29 mars 2019, p. 7.

Hague Kevin, Response to the Sustainable Growth Commission, These islands, juillet 2018 (www.theseislands.co.uk), consulté le 23 août 2018.

Herald, « Nicola Sturgeon Downplays Key Recommandation of the Growth Commission », 23 août 2018, p. 4.

HM Government, Scotland Analysis : Assessment of a Sterling Currency Union, Londres, Stationery Office, Cm 8815, février 2014. 
House of Commons (HC), Public Administration Select Committee, Lessons for Civil Service Impartiality from the Scottish Independence Referendum, Fifth Report, HC 111, 2015.

HC, Scottish Affairs Committee (SAC), Immigration and Scotland, Londres, Stationery Office, HC 488, juillet 2018.

HC, SAC, Scotland, Trade and Brexit, Londres, Stationery Office, HC 903, mars 2019.

Keating Michael, Harvey Malcolm, Small Nations in a Big World, Édimbourg, Luath Press, 2014.

Keating Michael, Is Small Always Beautiful ?, 31 mai 2018,

www.centreonconstitutionalchange.ac.uk/blog/small-always-beautiful, consulté le 15 janvier 2019.

Kerevan George, « Growth Report Gets an A+ for Effort, B- for Currency », The National, 26 mai 2018.

Leonard Richard, « Who Owns and Controls Scotland's Economy », in Bryan Pauline, Kane Tommy (dir), Class, Nation and Socialism, Glasgow, Glasgow Caledonia University, 2013, p. 16-22.

Leruez Jacques, Le Royaume-Uni, trente ans de difficultés, Paris, Hatier, Profil dossier, 1979.

Leruez Jacques, L'Écosse, une nation sans État, Lille, Presses universitaires de Lille, 1983.

Lineira Robert, Henderson Ailsa, Delaney Liam, « Voters' Response to the Campaign : Evidence from the Survey », in Keating Michael (dir.), Debating Scotland. Issues of Independence and Union in the 2014 Referendum, Oxford, Oxford University Press, 2017, p. 167-190.

McCrone Gavin, The Economics of Nationalism, Oxford, Blackwell, 1969.

McLaren John, « Reflections on the SNP's Sustainable Growth Commission Report », Scottish Trends, août 2018 (scottishtrends.co.uk), consulté le 10 septembre 2018.

Macwhirter Iain, Disunited Kingdom, Édimbourg, Cargo, 2014.

Migration Advisory Committee (MAC), EEA Migration in the UK : Final Report, Londres, septembre 2018.

Paluello Mika Minio, Jobs in the New Economy, Édimbourg, Scottish Greens, août 2015.

Progress Scotland, First Progress Scotland Poll Shows Brexit Impact, 30 mars 2019, www.progressscotland.org/first-progress-scotland-poll-shows-brexit-impact., consulté le 31 mars 2019.

Scottish Business UK, Win-Win. The Business Case for Scotland's Place in the UK, Édimbourg, septembre 2018.

Scottish Fiscal Commission (SFC), Scotland's Economic and Fiscal Forecasts, Édimbourg, mai 2018.

Scottish Government (SG), Government Expenditure and Revenue (GERS) 2009-2010, Édimbourg, février 2012.

SG, Nordic-Baltic Policy Statement, Édimbourg, mars 2014.

SG, GERS, 2012-2013, Édimbourg, février 2014.

SG, All Points North : the Scottish Government's Nordic-Baltic Policy Statement, Édimbourg, septembre 2017.

SG, Scotland's Place in Europe. People, Investment, Jobs, Édimbourg, janvier 2018.

SG, Scotland's Population Needs and Migration Policy : Discussion Paper on Evidence, Policy and Powers for the Scottish Parliament, Édimbourg, février 2018. 
SG, GERS, 2017-2018, Édimbourg, août 2018.

SG, Scotland's Place in Europe : An Assessment of the UK Government's Proposed Future Relationship with the EU, novembre 2018.

SG, No-Deal Brexit. Economic Implications for Scotland, Édimbourg, février 2019.

SG, A Trading Nation, A Place for Growing Scotland's Exports, Édimbourg, avril 2019.

Scottish Parliament (SP), Official Report (OR), 23 décembre 2016.

SP, OR, 21 janvier 2018.

SP, OR, 13 juin 2018.

SP, OR, 6 novembre 2018.

SP, OR, 16 janvier 2019.

SP, OR, 21 février 2019.

SP, OR, 8 mai 2019.

shafi Jonathan, « Independence Movement Must Fight Corporate Interests », The Herald, 25 avril 2019, p. 15.

Sheppard Tommy, « Thoughts on the Sustainable Growth Commission », Scottish Left Review, juillet 2018.

stuc, On Growth Commission Report, 25 mai 2018, www.stuc.org.uk/news/1352/growthcom, consulté le 20 mars 2019.

Sturgeon Nicola, Address to Conference, 28 avril 2019, www.snp.org/nicola-sturgeons-address-toconference, consulté le 29 avril 2019.

Sustainable Growth Commission (SGC), Scotland. The New Case for Optimism, Édimbourg, SNP, 2018.

Trebeck Katherine, Kerevan George, Boyd Stephen, Tackling Timorous Economics, Édimbourg, Luath Press, 2017.

Sources : SG, GERS, 2012-2013, Édimbourg, février 2014, p. 38, SG, GERS, 2017-2018, août 2018, chapitre 2 .

Source : SFC, Scotland's Economic and Fiscal Forecasts, Édimbourg, mai 2018, p. 91.

Source : HM Government, Scotland Analysis : Assessment of a Sterling Currency Union, Londres, mars 2014, p. 24.

Sources : SG, GERS 2009-2010, Édimbourg, février 2012, p. 6, 27 ; SG, février 2014, op. cit., p. 5 ; SG, août 2018, op. cit., introduction.

Sources : SG, février 2014, op.cit, p.5 ; SG, août 2018, op.cit, chapitre 1.

Source : Fraser of Allander Institute, Long-Term Economic Implications of Brexit, octobre 2016.

Source : SFC, op.cit, p. 84.

\section{NOTES}

1. LERUEZ Jacques, Le Royaume-Uni, trente ans de difficultés, Paris, Hatier, Profil dossier, 1979, p. 4. 
2. L'économiste Gavin McCrone avait publié une analyse pessimiste (MCCRONE Gavin, The Economics of Nationalism, Oxford, Blackwell, 1969, p. 106), avant de changer d'avis en 1974 dans une note confidentielle au gouvernement britannique.

3. FISCAL COMMISSION WORKING GROUP, The Economic Foundations of an Independent Scotland, Édimbourg, SG, août 2014. Le gouvernement britannique affina également ses analyses (programme Scotland Analysis).

4. LERUEZ Jacques, L'Écosse, une nation sans État, Lille, Presses universitaires de Lille, 1983, chapitre 5.

5. Sustainable Growth commission (SGC), Scotland. The New Case for Optimism, Édimbourg, SNP, 2018, 354 p. Wilson fut recruté par la Royal Bank of Scotland (RBS) en 1997, avant de fonder en 2014 un cabinet de conseil aux entreprises, Charlotte Partners. Militant au sein du SNP, il a travaillé pour Salmond dans les années quatre-vingt-dix, avant de siéger au Parlement écossais à ses débuts. Outre Jim Mather, ex-ministre des Entreprises de Salmond, sa commission comptait des universitaires (Catherine Schenk, Iain Docherty, Andrew Hughes Hallett), des chefs d'entreprises (Petra Wetzel, Marie Macklin, Mark Shaw, Dan McDonald, fondateur de N56, groupe de réflexion indépendantiste) et des élus au Parlement écossais (Kate Forbes et les ministres Derek MacKay, Shirley-Anne Sommerville), à la Chambre des Communes (Roger Mullin, battu en 2017) et dans une collectivité territoriale (Marie Burns).

6. Jacques Leruez avait d'ailleurs sollicité l'auteur de ces lignes, à deux reprises en 2012, pour évoquer les questions économiques écossaises dans le cadre de l'association franco-écossaise qu'il présidait.

7. MCLAREN John, « Reflections on the SNP's SGC Report », Scottish Trends, août 2018, 4.

8. PALUello Mika Minio, Jobs in the New Economy, Édimbourg, Scottish Greens, août 2015.

9. HAGUE Kevin, Response to the SGC, These islands, juillet 2018, 2. SP, OR, 13 juin 2018, col. 83, Adam Tomkins.

10. Derek MacKay, ministre des Finances et du Travail Équitable a renoncé à abaisser la taxe sur les passagers aériens, tout en incitant les collectivités territoriales à décourager l'usage de la voiture en ville. SP, OR, 8 mai 2019. Avec les compétences dévolues par le Scotland Act de 2012, le gouvernement écossais avait conservé une taxe sur les déchets non recyclés proche de celle du reste du royaume, et des capacités de recyclage insuffisantes.

11. SP, OR, 21 janvier 2018, Roseanna Cunningham, ministre de l'Environnement, du Changement climatique et de la Réforme foncière.

12. SGC, Scotland. The New Case for Optimism, op. cit., A5.

13. SP, OR, 16 janvier 2019, Dean Lockhart (Conservateur).

14. BeLl David, "The Contemporary Landscape », in GIBB Kenneth, MACLENNAN Duncan, MCNULTY Des, COMERFORD Michael (dir.), The Scottish Economy, Londres, Routledge, 2017, p. 29.

15. sGc, Scotland. The New Case for Optimism, op. cit, A6.129-156.

16. $S P, O R, 6$ novembre 2018, Neil Findlay (travailliste) relève que près de 500000 personnes en sont exclues.

17. Elle n'a pas été consultée par la SGC. STUC, On Growth Commission Report, 25 mai 2018.

18. Un million d'emplois écossais seraient menacés sur 15 ans. KEREVAN George, « Growth Report Gets an A+ for Effort, B- for Currency ", The National, 26 mai 2018, 2. Il préconise des expérimentations pour rompre avec les excès du capitalisme, TREBECK Katherine, KEREVAN George, BOYD Stephen, Tackling Timorous Economics, Édimbourg, Luath Press, 2017, p. 125.

19. MACWHIRTER Iain, Disunited Kingdom, Édimbourg, Cargo, 2014, p. 173.

20. En novembre 2018, le rapporteur spécial de l'ONU sur l'extrême pauvreté a reconnu leurs efforts, tout en pointant leurs limites dans le cadre existant. ALSTON Philip, Statement, Londres, novembre 2018. 
21. SP, OR, 21 février 2019, col. 41-54. Les Conservateurs sont obnubilés par les comparaisons avec l'Angleterre, pour l'impôt sur le revenu en 2018-2019 comme pour les droits de mutation à titre onéreux dévolus en 2012, SP, OR, 23 décembre 2016, col. 87, John Swinney, ministre des Finances.

22. SGC, Scotland. The New Case for Optimism, op. cit., B12.

23. MCLAREN John, op. cit., 5. Cet économiste, consulté par la SGC, estime que l'occasion doit être saisie.

24. SHAFI Jonathan, "Independence Movement Must Fight Corporate Interests ", The Herald, 25 avril 2019, p. 15. Shafi a co-fondé la Radical Independence Campaign en 2012 et le parti RISE après le référendum.

25. COMMON WEAL, White Paper Project. A Business Plan for a New Nation, Édimbourg, 2018, p. 20.

26. HERALD, "Nicola Sturgeon Downplays Key Recommandation of the Growth Commission ", 23 août 2018, p. 4. GRANT Alistair, "Row over Nats Plans for New Currency in an Independent Scotland ", The Herald, 5 mars 2019, p. 6.

27. STURGEON Nicola, Address to Conference, 28 avril 2019.

28. L'avis de Nicholas MacPherson, secrétaire permanent du Trésor, avait été rendu public, HC, PUBLIC ADMINISTRATION SELECT COMMITTEE, Lessons for Civil Service Impartiality from the Scottish Independence Referendum, 2015, 69.

29. MACWHIRTER Iain, Disunited Kingdom, op. cit., p. 173.

30. sGC, Scotland. The New Case for Optimism, op. cit., C3.25.

31. KEREVAN George, "Growth Report Gets an A+ for Effort, B- for Currency », art. cit., HAGUE Kevin, Response to the SGC, op. cit., 8.

32. SGC, Scotland. The New Case for Optimism, op. cit., C3.17. Afin de rassurer les marchés, la SGC propose une contribution écossaise annuelle au service de la dette britannique, fondée sur la proportion d'habitants, alors qu'en 2014, le gouvernement écossais envisageait une méthode de calcul plus favorable aux intérêts écossais, tout en conditionnant ces versements à l'obtention d'avantages dans les négociations sur l'indépendance.

33. SCOTTISH BUSINESS UK, Win-Win. The Business Case for Scotland's Place in the UK, Édimbourg, septembre 2018, p. 22. Le groupe est dirigé par Struan Stevenson, ancien député conservateur au Parlement européen.

34. Il fallait éviter d'obérer les capacités d'action du nouvel État. Tommy Sheppard, député SNP à la Chambre des Communes, s'en est félicité, SHEPPARD Tommy, "Thoughts on the SGC », Scottish Left Review, juillet 2018.

35. SGC, Scotland. The New Case for Optimism, op. cit., C2.6.

36. DALZELL Craig, Scottish Currency Options post-Brexit, Common Weal, juillet 2016.

37. Robin McAlpine (Common Weal), Colin Fox (SSP), ou encore Bella Caledonia. BELLA CALEDONIA, Replying to Andrew Wilson : a Scottish Currency is not Symbolic, it's Power, 14 février 2019.

38. BROоKS Lilly, "SNP Votes for Scotland to Quickly Adopt New Currency if it Leaves the UK», The Guardian, 27 avril 2019.

39. PROGReSS SCOTLAND, First Progress Scotland Poll Shows Brexit Impact, 30 mars 2019. John Curtice, politiste, s'est interrogé sur l'utilité de la réflexion menée par la SGC. CURTICE John, Does the Scottish Growth Commission Matter ?, 24 mai 2018.

40. SG, Nordic-Baltic Policy Statement, Édimbourg, mars 2014. SG, All Points North: the Scottish Government's Nordic-Baltic Policy Statement, Édimbourg, septembre 2017.

41. sGc, Scotland. The New Case for Optimism, op. cit., A.

42. Ibid., B7, A5.25.

43. Ibid., A6.212.

44. HAGUE Kevin, Response to the SGC, op. cit., 1, 2.

45. SP, OR, 13 juin 2018, col. 82, Neil Findlay. 
46. KEATING Michael, Is Small Always Beautiful ?, 31 mai 2018. Ce politiste a approfondi ces questions dans KEATING Michael, HARVEY Malcolm, Small Nations in a Big World, Édimbourg, Luath Press, 2014.

47. SGC, Scotland. The New Case for Optimism, op. cit., A3.

48. HC, SAC, Immigration and Scotland, Londres, Stationery Office, juillet 2018, 41, 79, 84, 98.

49. SG, Scotland's Place in Europe. People, Investment, Jobs, Édimbourg, janvier 2018, 24, 35, 43.

50. SG, Scotland's Population Needs and Migration Policy, Édimbourg, février 2018, 35, 131, 133.

51. SG, Scotland's Place in Europe: An Assessment of the UK Government's Proposed Future Relationship with the EU, Édimbourg, novembre 2018, 45.

52. HC, SAC, Scotland, Trade and Brexit, mars 2019, 33, George Hollingberry, ministre chargé de la politique commerciale. MAC, EEA Migration in the UK : Final Report, Londres, septembre 2018, 7.71.

53. EY, Sectoral Impact Analysis and British Readiness Assessment, Édimbourg, SG, janvier 2019.

54. SG, A Trading Nation, A Place for Growing Scotland's Exports, Édimbourg, avril 2019, p. 16.

55. C'est Richard Leonard, leader des Travaillistes écossais, qui regrette l'attribution, depuis 2007, du tiers des regional selective assistance à des entreprises non-écossaises. SP, OR, 16 janvier 2019. LEONARD Richard, "Who Owns and Controls Scotland's Economy », in BRYAN Pauline, KANE Tommy (dir.), Class, Nation and Socialism, Glasgow, Glasgow Caledonia University, 2013, p. 17.

56. sCOTTISH BUSINESS UK, Win-Win. The Business Case for Scotland's Place in the UK, op. cit., p. 25.

57. SG, No-Deal Brexit. Economic Implications for Scotland, Édimbourg, février 2019, p. 32-33. DHINGRA Swati, MACHIN Stephen, ovERMAN Henry, The Local Economic Effects of Brexit, Londres, London School of Economics, 2017.

58. Alyn Smith, député SNP au Parlement européen, cité par GRANT Alistair, «SNP Needs to Have Post-Brexit Answers Ahead of Indy Ref2 ", The Herald, 29 mars 2019, p. 7.

59. CARPENTER Griffin, «Troubled Waters, Scottish Fisheries and the EU », in HASSAN Gerry, GUNSON Russell (dir.), Scotland, the UK and Brexit. A Guide to the Future, Édimbourg, Luath Press, 2017, p. 75-76.

60. LINEIRA Robert, HENDERSON Ailsa, DELANEY Liam, « Voters' Response to the Campaign : Evidence from the Survey ", in KEATING Michael (dir.), Debating Scotland. Issues of Independence and Union in the 2014 Referendum, Oxford, Oxford University Press, 2017, p. 181.

61. DALZELL Craig, The Demographics of Independence, Common Weal, juin 2018. Seule la position des étrangers a connu une nette évolution en faveur de l'indépendance.

62. Selon Survation, en mars 2019, l'UE est devenue un enjeu aussi important que l'économie parmi les facteurs susceptibles d'orienter le vote sur l'indépendance ( $43 \%$ contre $22 \%$ en 2014), PROGRESS SCOTLAND, op. cit.

63. Colin Fox ou Stuart Campbell (Wings over Scotland). GORDON Tom, "Sturgeon Planning IndyRef2 Vote Before 2021 Ballot», The Herald, 25 avril 2019, p. 1. DALzelL Craig, "Scotland Deserves More than Trap of 'Soft Independence' », The Herald, $1^{\text {er }}$ mai 2019, p. 20. La stratégie du SNP est critiquée au sein même du parti (Joanna Cherry, députée à la Chambre des Communes).

\section{RÉSUMÉS}

Le SNP prône l'accession de l'Écosse à l'indépendance pour des raisons principalement économiques. Les responsables politiques écossais doivent pouvoir gérer les ressources naturelles et humaines pour stimuler la croissance et atteindre des finalités sociales. Cependant, 
lors du référendum d'autodétermination de 2014, nombre d'électeurs ont été dissuadés par des incertitudes persistantes, notamment au sujet de la devise. Un second référendum étant envisageable en réaction au Brexit, le SNP a créé la Sustainable Growth Commission pour formuler des propositions crédibles, démontrant la viabilité de l'Écosse et sa capacité à mener des politiques autonomes. Cet article examine le processus et les réactions suscitées jusqu'à l'adoption de la plupart de ses recommandations par le Congrès du parti en 2019.

The SNP wants Scotland to become independent chiefly on economic grounds. Scottish politicians ought to be able to run Scotland's natural and human resources to boost growth and meet social aims. Yet in 2014 when the referendum on Scotland's independence was held, there were lingering uncertainties - especially over the currency - which deterred many voters. As another referendum might be on the agenda in response to Brexit the SNP set up the Sustainable Growth Commission to devise credible responses proving Scotland's viability and its ability to conduct autonomous policies. This article examines the process and the reactions it triggered up to the 2019 SNP conference which endorsed most of its recommendations.

\section{INDEX}

Mots-clés : Écosse, indépendance, pétrole, monnaie, Brexit, Sustainable Growth Commission

Keywords : Scotland, independence, oil, Brexit, Sustainable Growth Commission

\section{AUTEUR}

\section{EDWIGE CAMP-PIETRAIN}

Université Polytechnique des Hauts-de-France (Valenciennes), CALHISTE EA4343 